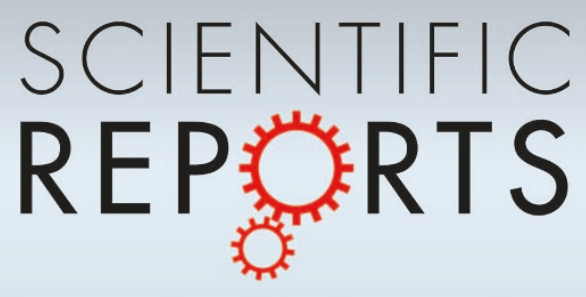

OPEN

SUBJECT AREAS:

ENDOCYTOSIS

RHO SIGNALLING

FLUORESCENCE IMAGING

CONFOCAL MICROSCOPY

Received

22 March 2013

Accepted

23 July 2013

Published

8 August 2013

Correspondence and requests for materials should be addressed to

N.A. (naraki@med.

kagawa-u.ac.jp)

* These authors contributed equally to this work.

† Current address:

Department of

Pharmacology, Faculty

of Medicine, Fukuoka

University, Fukuoka

814-0180, Japan.

\section{Dissecting the roles of Racl activation and deactivation in macropinocytosis using microscopic photo-manipulation}

\author{
Makoto Fujii*†, Katsuhisa Kawai*, Youhei Egami \& Nobukazu Araki
}

Department of Histology and Cell Biology, School of Medicine, Kagawa University, Miki, Kagawa 761-0793, Japan.

Macropinocytosis, a fluid-phase endocytosis, is a crucial pathway for antigen uptake and presentation in macrophages. We attempted to characterise the activation and deactivation of a small GTPase molecular switch, Racl, in macropinocytosis using microscopic photo-manipulation. Expression of genetically encoded photoactivatable-Rac1 (PA-Rac1) in RAW264 macrophages enabled the local, reversible control of macropinocytosis using blue laser irradiation. Marked membrane ruffling and unclosed

pre-macropinosomes were observed in the irradiated region of macrophages under the persistent activation of PA-Rac1. Although phosphatidylinositol 4,5-bisphosphate and actin were also localised to this region, the recruitment of maturating endosome markers, such as phosphatidylinositol 3-phosphate and Rab21, was restricted until PA-Rac1 deactivation. After deactivating PA-Racl by ceasing irradiation, membrane ruffling immediately receded, and the macropinosomes acquired maturation markers. These data suggest that activation of Racl is sufficient to induce membrane ruffling and macropinocytic cup formation, but subsequent deactivation of Racl is required for macropinosome closure and further maturation.

A ntigen-presenting cells, such as dendritic cells and macrophages, engulf extracellular fluid, including antigens, through macropinocytosis, a non-selective endocytic pathway ${ }^{1-5}$. Internalised antigens are processed, and antigenic peptides are loaded onto the major histocompatibility complex for T-cell presentation. Therefore, this pathway is critical to the immune system. In a paradoxical manner, numerous infectious pathogens, such as bacteria, viruses, protozoa and prions, utilise macropinocytosis for internalisation into host cells ${ }^{2-4}$. In addition, macrophage foam cell formation in atherosclerotic plaques can occur through the macropinocytic uptake of native low-density lipoprotein ${ }^{6}$. Given the physiological and pathological relevance of macropinocytosis, it is important to clarify the molecular mechanisms underlying this endocytic pathway in macrophages.

At the onset of macropinocytosis, actin-based membrane protrusions, termed lamellipodia or membrane ruffles, are preferentially formed at the cell periphery. Some membrane ruffles curve to form circular cup- or pocket-shaped structures. These open cups, or pockets, close and pinch off from the plasma membrane to form relatively large vesicles (greater than $0.2 \mu \mathrm{m}$ diameter), referred to as macropinosomes, within a few minutes after circular ruffle formation ${ }^{1,3,7,8}$. Internalised macropinosomes show centripetal movement and progressive maturation upon the acquisition of early and late endocytic components. Macropinosomes eventually fuse with lysosomes, although some of these structures are recycled back to the plasma membrane. The macropinocytic pathway is typically completed within $20 \mathrm{~min}$ from its initiation in macrophages, ${ }^{5,8,9}$.

The elucidation of the molecular basis of macropinosome formation and maturation is receiving increasing interest $^{1-4,10,11}$. The small GTPase Racl plays a crucial role in the formation of the initial membrane ruffles and macropinosomes. Activation of Rac1 induces actin polymerisation and subsequent lamellipodia formation in various cell types ${ }^{12,13}$. Overexpression of dominant active Racl (Rac1G12V) stimulates macropinocytosis in Rat-1 fibroblasts ${ }^{14}$. However, microinjection of dominant negative Rac1 (Rac1T17N) into immature dendritic cells inhibits macropinosome formation ${ }^{15}$. In addition, overexpression of a constitutively active mutant form of p21-activated kinase 1 (PAK1), a Rac1 effector, induces macropinosome formation, and overexpression of the PAK1 kinase autoinhibitory domain blocks both growth factor- and dominant active Rac1 (Rac1Q61L)-stimulated macropinocytosis ${ }^{16}$. However, these studies using dominant active or dominant negative mutants of Racl have not revealed how the activation/inactivation cycle of Racl affects macropinocytosis. Moreover, many experiments have focused on growth factor stimulation, which simultaneously induces numerous intracellular 
signalling pathways; therefore, it is unknown whether the activation of Rac1 is sufficient to induce macropinosome formation.

Recently, Yoshida et al. investigated the relationship between Rac1 activation and macropinosome formation using ratiometric and fluorescence resonance energy transfer (FRET) microscopy in macrophages stimulated with macrophage colony-stimulating factor (M-CSF) and revealed that Racl is transiently activated during macropinosome formation ${ }^{7}$. The obtained FRET images implied that the spatiotemporal activation followed by deactivation of Rac1 might be important for coordinated process of macropinosome formation and maturation, although the significance of Racl deactivation has not been determined.

To analyse the precise role of the spatiotemporal activation of Rac1, which is a key regulator of actin polymerisation in cell migration $^{12,13}$, Wu et al. established photoactivatable-Rac1 (PA-Rac1), which is a fusion of a dominant active Racl with the light oxygen voltage (LOV) domain and following carboxy-terminal helical extension $(\mathrm{J} \alpha)$ of phototropin 1, a protein light switch found in Avena sativa ${ }^{17,18}$. In the dark, the flavin-binding LOV domain interacts with $\mathrm{J} \alpha$ and sterically hinders the interaction between Racl and downstream effectors. Blue laser irradiation induces the dissociation of the LOV domain and $\mathrm{J} \alpha$ to activate Racl. This photo-induced activation is reversible. Using this system, Wu et al. demonstrated that localised Rac activation was sufficient to generate cell motility and regulate the direction of cell movement ${ }^{17,18}$.

To clarify the roles of Racl activation and deactivation in the coordinated process of macropinocytosis, we introduced PA-Racl into RAW264 macrophages. Photo-manipulation of PA-Racl using a 445-nm laser enabled local control of membrane ruffling and macropinocytosis in live macrophages. Using this system, we demonstrated that markers of initial membrane ruffling and macropinocytic cups, such as phosphatidylinositol 4,5-bisphosphate $\left(\mathrm{PI}(4,5) \mathrm{P}_{2}\right)$ and actin, were detected upon activation of PA-Rac1. However, the recruitment of maturating macropinosome markers was observed after the deactivation of PA-Racl. In this study, we have emphasized the importance of spatiotemporal regulation of Rac1 activation/deactivation for the progress of macropinocytosis.

\section{Results}

Local activation of PA-Racl reversibly induces membrane ruffling and macropinocytic cup formation. Several studies have suggested that Rac1 functions in membrane ruffling and macropinocytosis; however, these studies primarily involved the use of dominant active or negative mutants of $\mathrm{Racl}^{14,15}$. To elucidate the relationship between Racl activation/inactivation cycle and macropinosome formation, we introduced mCherry-fused PA-Rac1 into RAW264 macrophages. After confirming the expression of mCherry-PARacl based on mCherry signals, we irradiated the peripheral region of the cells using a 445-nm laser during intervals of image acquisition and acquired phase-contrast images of live cells every $15 \mathrm{sec}$ using confocal microscopy. Photoactivation of PA-Racl using a 445-nm laser led first to cell surface membrane ruffling within a minute and then to formation of phase-bright bubble-like structures, which were presumably macropinocytic cups or macropinosomes, at the site of irradiation (Fig. 1a and Supplementary Movie 1). Typically, several bubble-like structures were formed $3 \mathrm{~min}$ after beginning photoactivation. The number of bubble-like structures increased with time during photoactivation (Supplementary Table S1). Depending on the individual cells, the initial membrane ruffles formed 1-5 min after photoactivation. This time lag might reflect the expression level of mCherry-PA-Rac1 or other downstream effector molecules of Rac1 in cells. We have tried different irradiation area sizes ranging from 50 to $200 \mu \mathrm{m}^{2}$. However, the relationship between the irradiation area size and the number of bubble-like structures was undefined, since the number of formed bubble structures considerably varies from cell to cell. As for the location of irradiation, ruffling and bubble structures were formed only when the cell peripheral region was photoactivated. We could not see such structures in the cell central region upon photoactivated, although the reason remains unknown.

After ceasing irradiation, the number of bubble-like structures was gradually reduced (Supplementary Table S1). Although numerous numbers of bubble-like structures $(58.8 \pm 6.8, \mathrm{n}=6)$ had been accumulated in the cell peripheral region photoactivated for $15 \mathrm{~min}$, a limited number of phase-bright vesicles $(8.7 \pm 3.1, \mathrm{n}=$ 6) remained 15 min after turning off PA-Pacl activation (Fig. $1 \mathrm{~b}$ and Supplementary Movie 2, Supplementary Table S1). Many other bubble-like structures disappeared, probably by ruffle receding and/or vesicle recycling back to the plasma membrane as previously reported ${ }^{5,8}$.

To confirm whether these phenomena were photoactivation dependent and repeatable, we moved the irradiation spot to a different position in the same cell. The membrane ruffles immediately receded from the initial irradiation area, but spherical vacuoles remained, and membrane ruffles were formed in the newly irradiated area (Fig. 1c and Supplementary Movie 3). Upon PA-Rac1 photoactivated, it was found that the local induction of membrane ruffling was accompanied by increased fluorescence signals of mCherry-PARac1. After the irradiation moving out, PA-Rac1 was gradually dissociated from the membranes of the spherical macropinosome-like structures (Fig.1c and Supplementary Movie 3).

Overexpression of a GDP-bound (dominant negative) mutant of PA-Rac1 (PA-Rac1 T17N) did not induce marked membrane ruffling or the formation of bubble-like structures after 445-nm laser irradiation (Supplementary Fig. S1 and Supplementary Movie 4). This finding indicated that the morphological changes induced by 445-nm laser irradiation are dependent on GTP-loaded Rac1.

Deactivation of Racl is required for efficient macropinosome closure. To determine whether the bubble-like structures formed by PA-Rac1 photo-manipulation are opened cups or closed vesicles, we added fluorescein dextran (FDx), a fluid-phase probe, to the extracellular medium while photoactivating and incubated for $5 \mathrm{~min}$, and then washed out with cold PBS. When we did not turn off the 445-nm laser irradiation until FDx was washed out of the medium, FDx was faintly incorporated into intracellular vesicles (Fig. 2a). Since FDx was released from bubble-like structures, it is likely that these structures were not closed. However, when we turned off the $445 \mathrm{~nm}$ irradiation 2 min before washing out, FDx remained in macropinosomes; suggesting that these macropinosomes were closed after ceasing the photoactivation (Fig. 2b). Furthermore, we quantified the rate of closed macropinosmes at different time points $(0 \mathrm{~min}, 0.5 \mathrm{~min}, 2 \mathrm{~min}$ and $5 \mathrm{~min}$ ) after turning off the PA-Racl photoactivation. After confirming the formation of bubble-like structures by PA-Rac1 activation, we added cold PBS containing FM4-64 lipophilic dye (which exhibits red fluorescence upon binding to the outer leaf of the plasma membrane) to the extracellular medium without ceasing irradiation (at time point $0 \mathrm{~min}$ ). In this case, most of the bubble-like structures were labelled with the FM4-64 dye, suggesting that these structures maintained a connection with the plasma membrane (Fig. 2c). Quantitation of bubble-like structures unlabelled with the FM dye indicated that only $6.5 \pm 1.8 \%(n=6)$ of bubble-like structures were closed macropinosmes (Fig. 2d). However, when we added the FM4-64 dye at $0.5 \mathrm{~min}$ after ceasing irradiation, some vacuoles $(35.4 \pm 5.4 \%, \mathrm{n}=9$ ) were inaccessible to the FM 464 dye, suggesting that these structures are closed intracellular macropinosomes before adding the FM dye. The percentage of closed macropinosomes significantly $(\mathrm{P}<0.05$, Student's $t$-test $)$ increased $0.5-2 \mathrm{~min}$ after ceasing the irradiation (Fig. $2 \mathrm{~d}$ ). It was shown that $83.9 \pm 6.5 \%(\mathrm{n}=6)$ of bubble-like structures were closed into macropinosomes $5 \mathrm{~min}$ after PA-Racl deactivation. (Fig. 2c, d). Taken together, these data indicate that activation of 
(a)
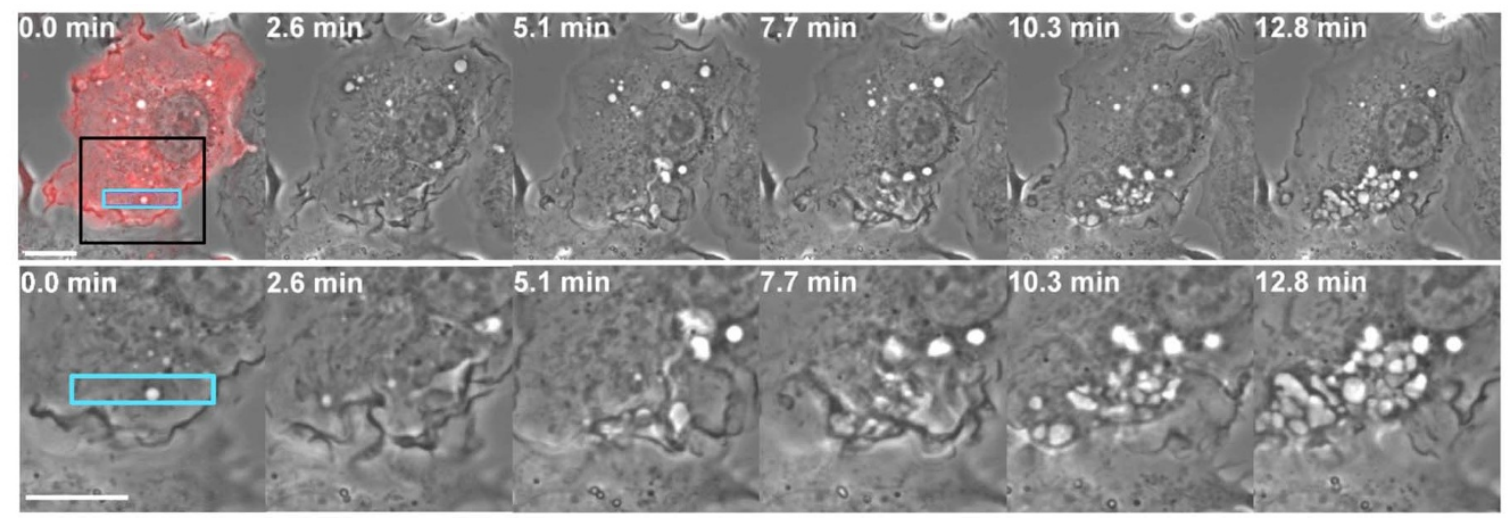

(b)

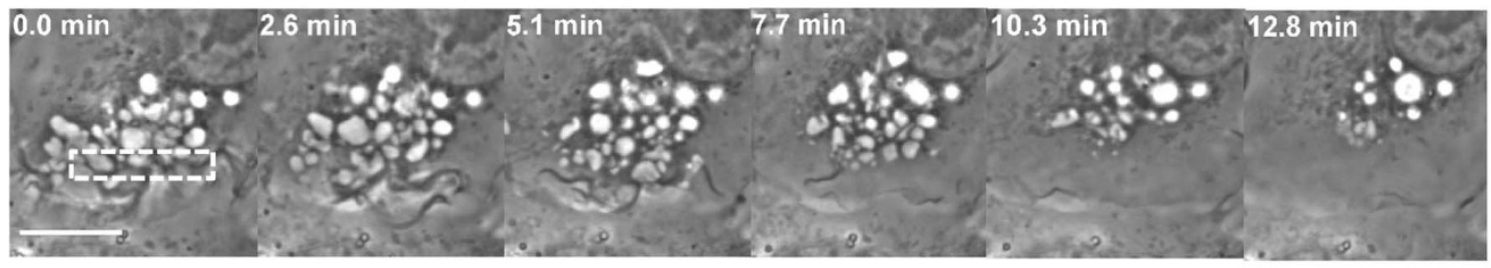

(c)
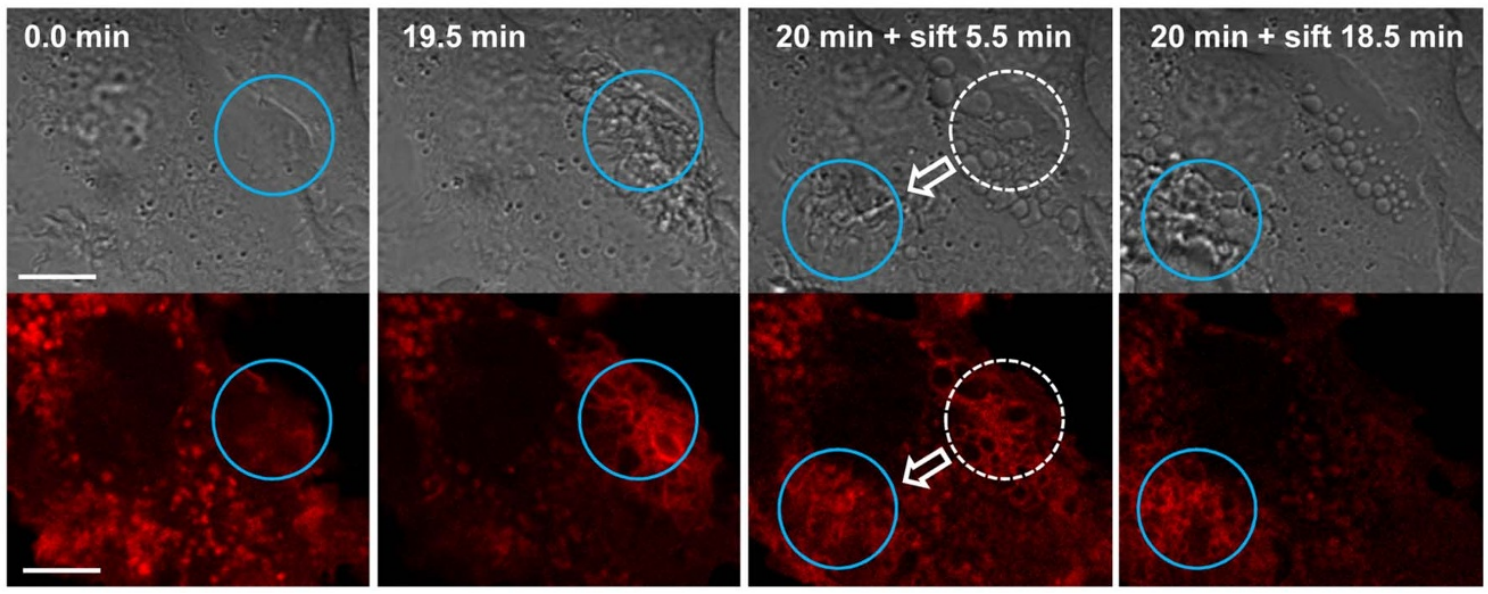

Figure 1 Photo-manipulation of PA-Rac1 reversibly controls membrane ruffling and macropinosome formation. (a) Time-lapse imaging of RAW264 cells expressing mCherry-PA-Rac1 during photoactivation using $445 \mathrm{~nm}$ laser irradiation. RAW264 cells were transiently transfected with pTriEx/ mCherry-PA-Racl and subjected to local photoactivation of PA-Racl (blue rectangular area). The first panel shows a merged image of phase-contrast and mCherry fluorescence before the photoactivation of PA-Rac1, confirming the expression of mCherry-PA-Racl. The elapsed times after starting the irradiation are shown at the top of each panel. The lower panels show magnified images of the boxed area of the cell. The bar indicates $10 \mu \mathrm{m}$. (b) Timelapse series of the same region of the cell shown in Fig. 1a after ceasing irradiation. Elapsed times after ceasing the irradiation are shown at the top of each panel. The bar indicates $10 \mu \mathrm{m}$. (c) DIC (upper) and mCherry (lower) images of the mCherry-PA-Racl expressing cell irradiated at two different positions sequentially. Time 0 indicates the initiation of blue laser irradiation in the area enclosed by a blue circle (the first panel). At 19.5 min, ruffles were apparent within the irradiated region (blue circle in the second panel). After $20 \mathrm{~min}$, the irradiation was shifted to a different area of the same cell. After ceasing the irradiation, the ruffles immediately receded, and spherical macropinosomes were formed in the initial irradiation area (broken-lined circle in the third panel). Instead, marked ruffling was induced in the new irradiated area (blue circles in the third and fourth panels). The bar indicates $10 \mu \mathrm{m}$.

PA-Racl is sufficient for the formation of membrane ruffles and bubble-like structures, which are connected to the cell surface, and subsequent PA-Rac1 deactivation is required for the efficient closure of macropinosomes.

Because we found that the bubble-like structures are unclosed macropinocytic cups/pockets, we hereafter refer to these structures as pre-macropinosomes.

Ultrastructural characterisation of the phenotype of persistent activation of Rac1. To confirm the phenotype of persistent Rac1 activation, RAW264 cells were transfected with pCitrine-Rac1Q61L, a constitutively active Rac1 mutant, and serum-starved in RB for 2 hours before observation. By light microscopy, it was found that the Citrine-Rac1Q61L-expressing cells exhibited marked cell surface ruffling and bubble-like structures, while cells not expressing CitrineRac1Q61L lost membrane ruffles after 2-hour serum starvation (Supplementary Fig. S2). When FM4-64 diluted in cold PBS was added to the extracellular medium, the membranes of the cell surface ruffles and bubble-like structures were immediately labelled with the lipophilic dye (Fig. 3a). Therefore, these bubble-like structures seemed to be identical to the unclosed pre-macropinosomes observed in Fig. 2c of which PA-Racl was photoactivated.

To further characterise the ultrastructural phenotype of persistent activation of Rac1, RAW macrophages expressing CitrineRac1Q61L were fixed with 4\% glutaraldehyde and processed for scanning electron microscopy (EM) as previously described ${ }^{11}$. Based on the cell morphology observed by light microscopy (Supplementary Fig. S2), Rac1Q61L expressing cells with numerous 

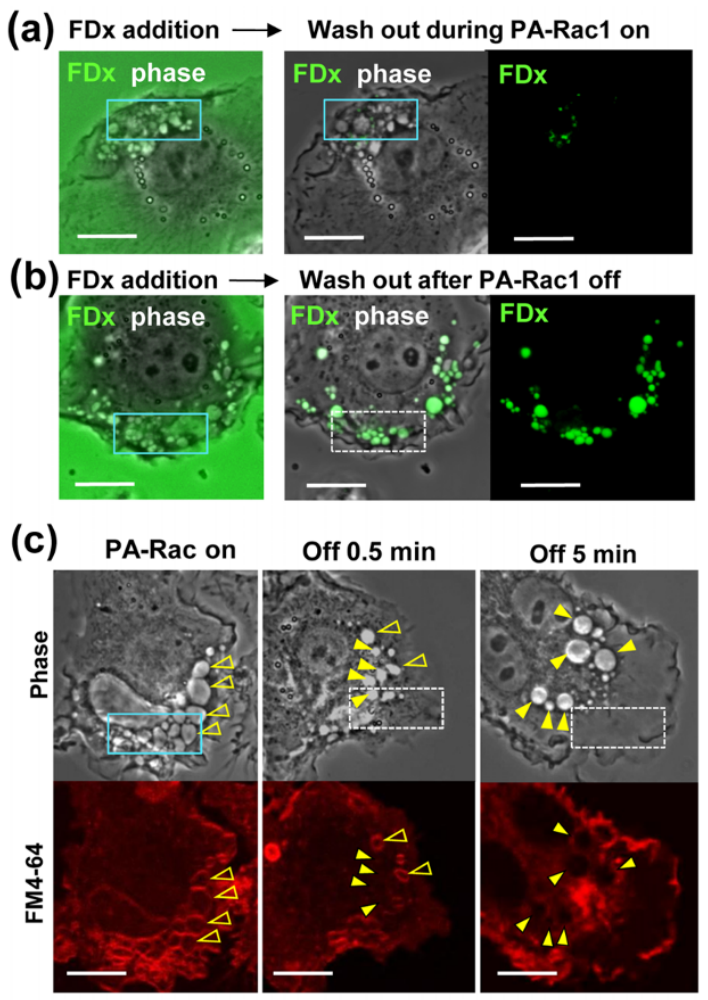

(d)

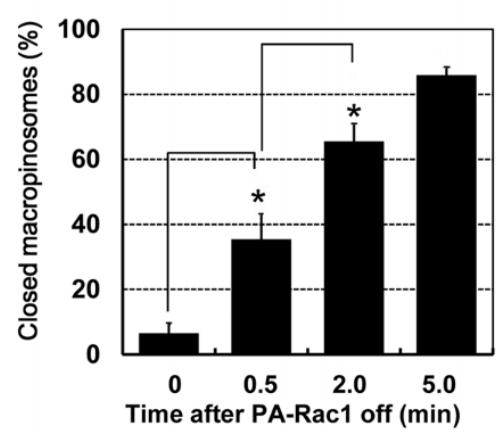

Figure $2 \mid$ The deactivation following activation of PA-Rac1 is required for the closure of macropinosomes. (a) We added FDx, a fluid-phase probe, to the extracellular medium while photoactivating and incubate for 5 min followed by washing with cold PBS. Then, phase-contrast and FDx images were immediately acquired. Photoactivation was not turned off until washing out FDx. The left panel is a merged image of FDx and phasecontrast before washing out FDx. The middle (FDx, Phase merged) and right (FDx only) images were acquired immediately after washing out. Of note, FDx was largely released from the bubble-like structures by washing out. (b) FDx was added and incubated for $5 \mathrm{~min}$ as the same as Fig.2a. Photoactivation was turned off 2 min before washing out FDx with PBS. A significant amount of FDx was observed in bubble-like structures after washing out. (c) After confirming bubble-like structures by the PA-Rac1 activation, FM4-64 lipophilic dye was added to the extracellular medium at different time points of $0 \mathrm{~min}$ (PA-Rac on), $0.5 \mathrm{~min}$, and $5 \mathrm{~min}$ after turning off the photoactivation. The phase-contrast (upper) and FM4-64 fluorescence (lower) images were acquired within 1 min after the FM 4-64 addition. The membrane of unclosed pre-macropinosomes can be labelled with FM4-64 (open arrowheads at $0 \mathrm{~min}$ and $0.5 \mathrm{~min}$ ), but closed macropinosomes cannot be labelled with FM4-64 (filled arrowheads at $0.5 \mathrm{~min}$ and $5 \mathrm{~min}$ ). The bar indicates $10 \mu \mathrm{m}$. (d) Quantitation of macropinosome closure after PA-Racl deactivation. By repeating the experiments shown as Fig.2c, the percentage of FM4-64-negative closed macropinosomes in the total of phase-bright macropinocytic structures was calculated. The data presented are the mean \pm standard error of four independent experiments. ${ }^{*} \mathrm{p}<0.05$, Student's $t$-test.

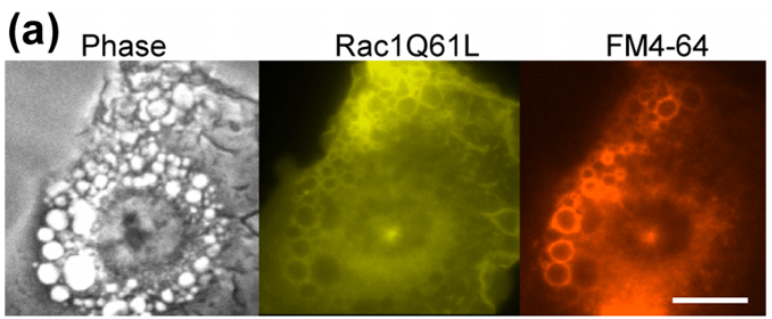

(b)
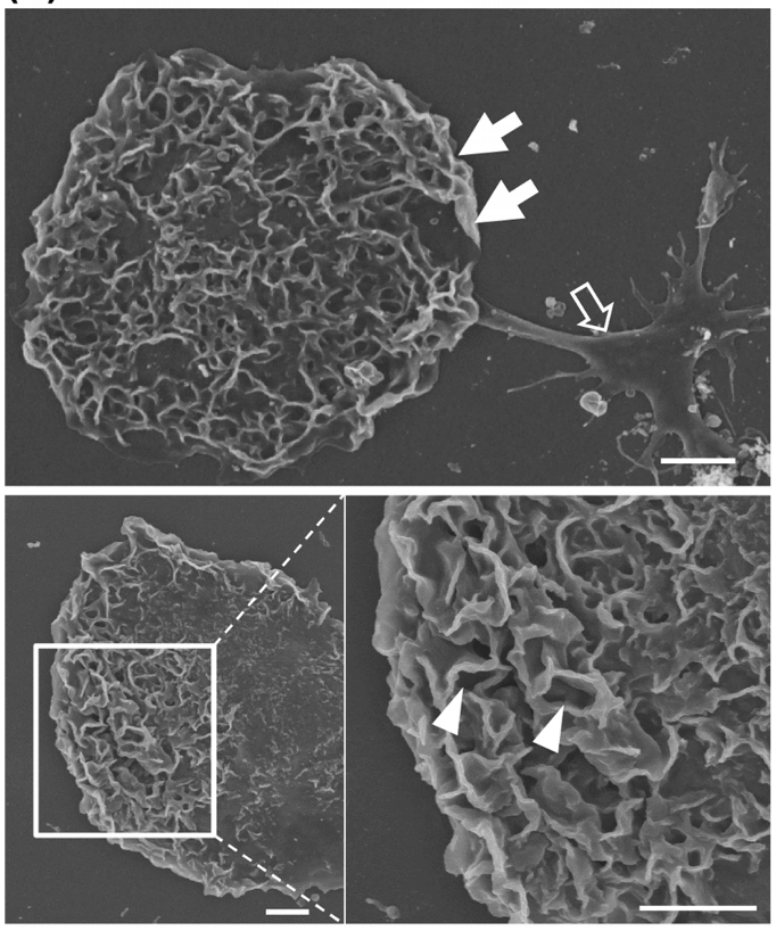

Figure $3 \mid$ Observation of RAW264 cells expressing constitutively active mCitrine-Rac1Q61L by fluorescence and scanning electron microscopy. RAW264 cells were transfected with pmCitrine-Rac1Q61L. After 12 hours, the cells were serum starved for 2 hours and then subjected to live-cell imaging or processed for scanning EM. (a) After confirming the expression of mCitrine-Rac1Q61L by fluorescence microscopy, we added FM4-64 to the extracellular medium and immediately $(<1 \mathrm{~min})$ acquired phasecontrast, mCitrine-Rac1Q61L and FM4-64 images. RAW264 cells expressing mCitrine-Rac1Q61L showed remarkable membrane ruffling and phase-bright vacuoles. Most of the phase-bright vacuoles associated with mCitrine-Rac1Q61L (yellow) were labelled with FM4-64 (red), indicating that these structures are unclosed pre-macropinosomes. The bar indicates $10 \mu \mathrm{m}$. (b) Scanning EM of RAW264 cells expressing constitutively active Rac1Q61L. We can identify the cell showing numerous ruffles across the whole dorsal surface as a Rac1Q61L expressing cell (arrows), since non-expressing cells lost dorsal ruffles and lamellipodia (open arrow) as observed by fluorescence microscopy (Supplementary Fig. S2). A higher magnification of the ruffling region shows the openings of macropinocytic cups or pockets (arrowheads). The bars indicate $5 \mu \mathrm{m}$.

cell surface ruffles could be clearly distinguished from cells not expressing this mutant, which showed a loss of ruffles after 2-hour serum starvation (Fig. 3b, upper). In Rac1Q61L expressing cells, multiple invaginations or caves were observed among the ruffles using scanning EM (Fig. 3b). The opening of these caves at the cell surface suggests a failure of macropinosome closure under persistent Racl activation.

Recruitment and activation of downstream effectors are locally induced after PA-Rac1 photoactivation. Next, we co-expressed 
mCherry-PA-Rac1 with Citrine-PBD, the p21-binding domain from PAK1, which only binds activated $\mathrm{Racl}^{7}$. Upon laser irradiation at $445 \mathrm{~nm}$, we detected accumulation of Citrine-PBD around the irradiated area where the formation of membrane ruffles and premacropinosomes was observed (Fig. 4a). These findings indicate that irradiation substantially activated PA-Rac1 to bind downstream effectors and recruit effector molecules to the region.

Phosphoinositides, such as $\mathrm{PI}(4,5) \mathrm{P}_{2}$, are key regulators of the actin cytoskeleton and membrane trafficking ${ }^{19-21}$. We have previously shown that $\mathrm{PI}(4,5) \mathrm{P}_{2}$ is predominately localised to membrane ruffles and macropinocytic cups ${ }^{22}$. Because Racl activates phosphatidylinositol 4-phosphate 5-kinase (PIP5K), which produces $\mathrm{PI}(4,5) \mathrm{P}_{2}{ }^{23,24}$, the level of $\mathrm{PI}(4,5) \mathrm{P}_{2}$ could be elevated upon the PARacl activation. To confirm this possibility, we co-transfected
mCherry-PA-Rac1 with a YFP-fused phospholipase C- $\delta_{1}$ pleckstrin homology domain (YFP-PLC-PH), which specifically binds $\mathrm{PI}(4,5) \mathrm{P}_{2}$. As expected, live-cell imaging of YFP-PLC-PH demonstrated increased $\mathrm{PI}(4,5) \mathrm{P}_{2}$ levels in membrane ruffles and premacropinosomes during PA-Rac1 photoactivation (Fig. $4 \mathrm{~b}$ and Supplementary Movie 5).

Actin assembly and disassembly can be controlled by photomanipulation of PA-Racl. Actin reorganisation is an essential step in the formation of membrane ruffles and macropinosomes ${ }^{1-4,12,13}$. To analyse actin dynamics during PA-Racl-induced membrane ruffling and macropinosome formation, we co-expressed mCherry-PA-Rac1 with YFP- $\beta$-actin. We observed that YFP- $\beta$-actin was predominantly associated with the membrane ruffles and pre-macropinosomes

\section{(a)}

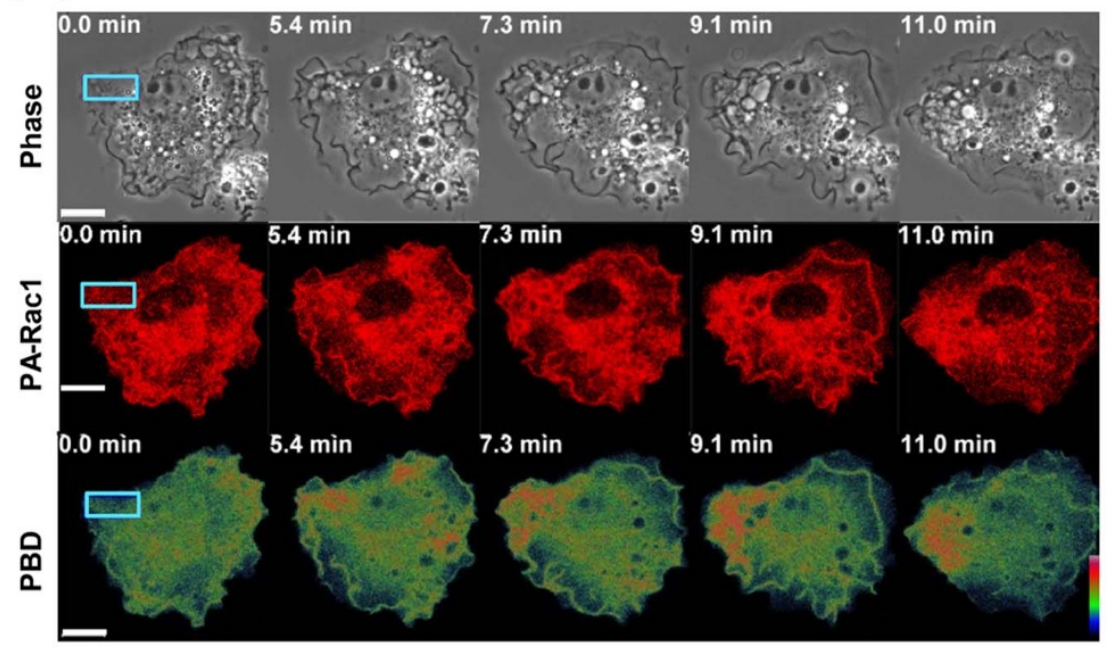

(b)

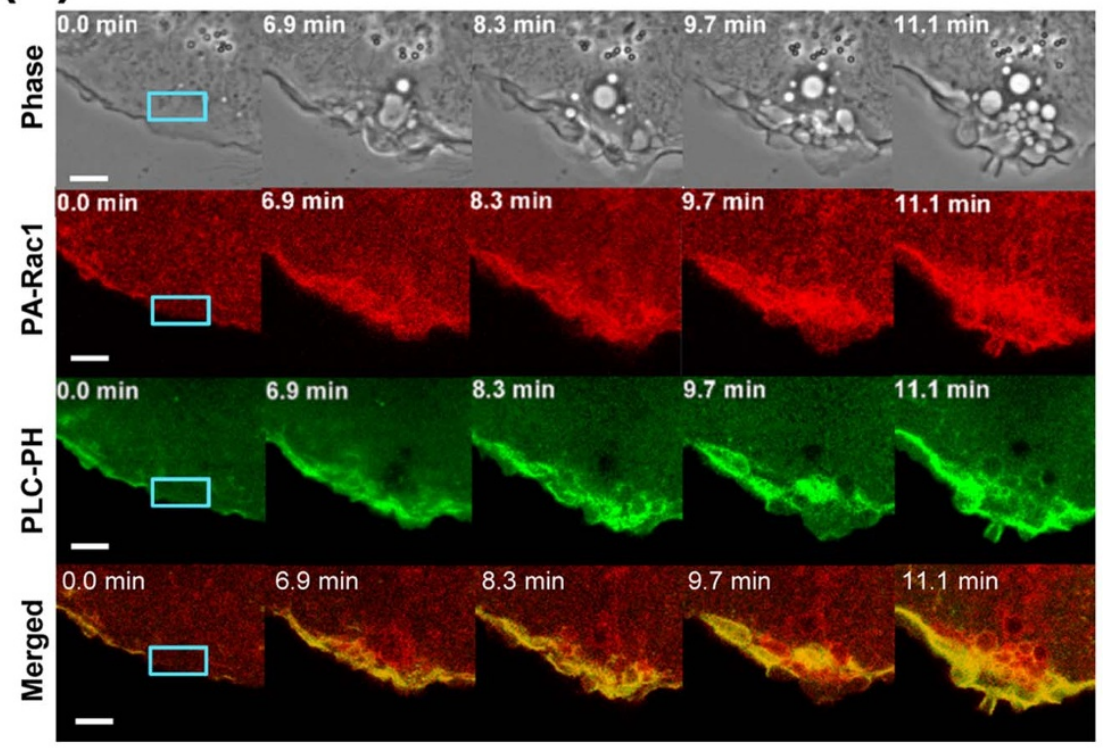

Figure $4 \mid$ Local PA-Rac1 activation recruits the PBD of Pak1 and induces PI(4,5) $\mathrm{P}_{2}$ production. (a) Recruitment of the Citrine-p21-binding domain (PBD) of Pak1 to the photoactivated region. RAW264 cells were co-transfected with pTriEx/mCherry-PA-Rac1 and pCitrine-PBD. The laser-irradiated area is indicated with a blue rectangle. The selected time-lapse images of phase-contrast, mCherry-PA-Racl(red) and Citrine-PBD (signal intensity is shown by spectrum colour), captured at the indicated times were shown. Time 0 indicates the initiation of photoactivation. The bar indicates $10 \mu \mathrm{m}$. (b) The level of $\mathrm{PI}(4,5) \mathrm{P}_{2}$ was locally increased in the region where membrane ruffles and pre-macropinosomes were formed during photoactivation. RAW 264 cells were co-transfected with pTriEx/mCherry-PA-Racl and pYFP-PLC- $\delta_{1}$ PH. The selected time-lapse images of phase-contrast, mCherry-PA-Racl (red),YFP-PLC- $\delta_{1}$ PH (green) and merged images captured at the indicated times were shown. The laser-irradiated area is indicated with a blue rectangle. Time 0 indicates the initiation of photoactivation. The bar indicates $5 \mu \mathrm{m}$. 
(Fig. 5a and Supplementary Movie 6). Quantification of the YFP signal intensity confirmed the accumulation of YFP- $\beta$-actin in the photoactivated area (Fig. $5 \mathrm{c}$ ). After ceasing blue laser irradiation to deactivate PA-Racl, the membrane ruffles and YFP- $\beta$-actin signal disappeared from this area (Fig. 5b, c and Supplementary Movie 7). Thus, actin assembly and disassembly can be controlled by the local photo-manipulation of PA-Rac1, probably through the production of $\mathrm{PI}(4,5) \mathrm{P}_{2}$ and actin-binding proteins.

$\mathrm{PI}(3) \mathrm{P}$ is scarcely recruited to areas where PA-Rac1 is persistently activated. Internalised macropinosomes sequentially acquire early and late endosome markers and eventually fuse with tubular lysosomes in macrophages ${ }^{9,25,26}$. Phosphatidylinositol 3-phosphate (PI(3)P) primarily localises to early endosomes ${ }^{20,21}$ and serves as a binding platform for early endosome proteins, such as early endosome antigen 1 (EEA1) and sorting nexins through FYVE and Phox homology (PX) domains, respectively ${ }^{3}$. During the normal course of macropinocytosis, PI(3)P accumulates on macropinosomes after 10$20 \mathrm{~min}^{7,27,28}$. To analyse the impact of PA-Racl activation on PI(3)P levels, we overexpressed mCherry-PA-Racl with Citrine-FYVE, which specifically detects PI(3)P. Prior to PA-Racl photoactivation, Citrine-FYVE was primarily localised on small early endosomes. During persistent activation of PA-Racl by repeated blue laser irradiation, many pre-macropinosomes $(60.5 \pm 7.8, \mathrm{n}=6)$ accumulated in the irradiated area; however, $89.1 \pm 3.5 \%(n=6)$ of these structures were negative for Citrine-FYVE even after $20 \mathrm{~min}$ (Fig. 6a and Supplementary Movie 8), suggesting that the persistent activation of PA-Rac1 does not induce elevation of PI(3)P levels.

Curiously, after transient activation of PA-Rac1 by a 5-min pulse of blue laser irradiation, newly formed macropinosomes efficiently acquired PI(3)P (Fig. 6b and Supplementary Movie 9). The number of pre-macropinosomes formed by 5 -min pulse photoactivation was much less $(22 \pm 7.1, \mathrm{n}=4)$ than that by 20 -min persistent photoactivation, however, $75.2 \pm 12.1 \%(\mathrm{n}=4)$ of formed pre-macropinosmes became positive for Citrine- FYVE $10 \mathrm{~min}$ after ceasing photoactivation, similar to the normal course of macropinocytosis. This finding implies that Racl deactivation after transient activation of Rac1 is important for the maturation of early macropinosomes.

The deactivation of Racl is crucial for the progress of macropinosome maturation or recycling. We have previously demonstrated that Rab21 predominantly localises to macropinosomes from early Rab5-positive to late Rab7-positive stages, and active Rab21 is not required for macropinosome formation, but implicated primarily in macropinosome maturation ${ }^{25}$. Since GFP-Rab21 was more predominantly localised to macropinosmes than Rab5 or Rab7 $7^{25}$, we employed Rab21 as a marker of macropinosome maturation to determine the significance of Racldeactivation in macropinosome maturation. While photoactivation of PA-Racl induced premacropinosome formation in cells co-expressing PA-Racl and GFP-Rab21, few of them $(6.4 \pm 1.8 \%, \mathrm{n}=6)$ were GFP-Rab21positive under the persistently active $(>15 \mathrm{~min})$ of PA-Racl (Fig. 7a and Supplementary Movie 10). When we ceased photoactivation of PA-Racl, the total number of pre-macropinosomes and/ or macropinosomes was reduced with time. At this stage, many of the pre-macropinosomes receded without forming macropinosomes. Some of the sustained macropinosomes migrated toward the perinuclear region, and these motile macropinosomes showed membrane localisation of GFP-Rab21 signals (Fig. 7b and Supplementary Movie 11). In contrast, the percentage of Rab21-positive macropinosomes markedly increased $(78.6 \pm 6.8 \%, n=6)$ after $10 \mathrm{~min}$. These data suggest that deactivation of Racl may be required for the transition from pre-macropinosomes to maturating macropinosome.

\section{Discussion}

Many previous studies have used dominant active or negative mutants of Rac to elucidate the relationship between Rac activity and cellular events ${ }^{14,15}$. However, these mutants are not suitable tools for analysing the physiological function of Rac1 as a molecular switch, since the effects of mutant expression are irreversible and global in the cell. Therefore, we utilised PA-Rac1, which is reversibly activated by blue laser irradiation ${ }^{17,18}$, to investigate the relationship between the activation/deactivation of Racl and macropinosome formation and maturation. Using this advantageous technique, we were locally able to induce membrane ruffling and macropinosome formation. Markers of initial membrane ruffle and macropinocytic cup formation, such as $\mathrm{PI}(4,5) \mathrm{P}_{2}$ and actin $^{22}$, were detected on these structures before PA-Rac1 deactivation, but acquisition of Rab21, a macropinosome maturation marker ${ }^{25}$, was observed only after Rac1 deactivation. Moreover, the persistent activation of PA-Rac1 suppressed macropinosome internalisation and induced the accumulation of pre-macropinosomes at the cell periphery, where laser irradiation was repeatedly applied for more than $20 \mathrm{~min}$. Taken together, these data suggest that activation of Rac1 is sufficient for membrane ruffles and macropinocytic cups to form, whereas deactivation of Racl is required for the efficient closure of macropinosomes and further maturation and/or recycling.

Using FRET microscopy, Yoshida et al. vigorously investigated the timing of Racl activation during macropinosome formation and maturation in M-CSF-stimulated macrophages ${ }^{7}$. They divided macropinocytosis into four stages based on cell morphology, characterised by the formation of irregular ruffles, curved ruffles, circular ruffles and motile macropinosomes, and showed that Racl is transiently activated after circular ruffle formation. Based on these data, these authors concluded that transient Racl activation induces cup closure for isolated macropinosome formation. Our data consistently showed that the deactivation of Racl following activation was important for efficient cup closure. Our fluid-phase uptake observations using FDx and quantitation of macropinosome closure using FM4-64 membrane labelling (Fig. 2a-d) clearly revealed that the bubble-like macropinocytic structures formed under the persistent activation of PA-Rac1 were open to extracellular medium, whereas after deactivation of Rac1, these structures closed to form intracellular vesicles.

Inconsistent with the data obtained in the present study, it was previously shown that a constitutively active mutant of Racl enhanced macropinosome formation ${ }^{14}$. However, this previous study did not address the effect of the deactivation of Rac1. Our analysis of RAW macrophages overexpressing constitutively active Racl by fluorescence microscopy using FM4-64 dye and scanning EM revealed that the bubble-like structures are primarily pre-macropinosomes connected to the cell surface. This phenotype is similar to that of PA-Rac1-expressing cells when persistently photoactivated.

During constitutive or growth factor-induced macropinocytosis, macropinosomes are sustained for minutes and gradually diminishes associated with homotypic fusion and/or recycling back to the plasma membrane $e^{7,822,27-29}$. In contrast, under the persistent activation of PA-Rac1, many pre-macropinosomes accumulate over time at the cell periphery, and few of them acquired the early endosome markers PI(3)P and Rab21. Then after the deactivation of PA-Rac1, pre-macropinosomes gradually disappeared, and a population of these structures acquired Rab21. These Rab21-positive vesicles were typically sustained for a long period, showing centripetal movement toward the perinuclear region. This population might reflect maturating macropinosomes. This finding is consistent with previous results showing that some macropinosomes mature, while others are recycled back to the plasma membrane ${ }^{5,8-10,22}$. These data also suggest that deactivation of Racl is required not only for the closure of macropinosomes, but also for the recruitment of molecules which may mediate the maturation of macropinosomes.

What are the upstream activators and the downstream effectors of Racl during the macropinosome formation under physiological condition? It has been suggested that the receptor tyrosine kinase-mediated 
(a)

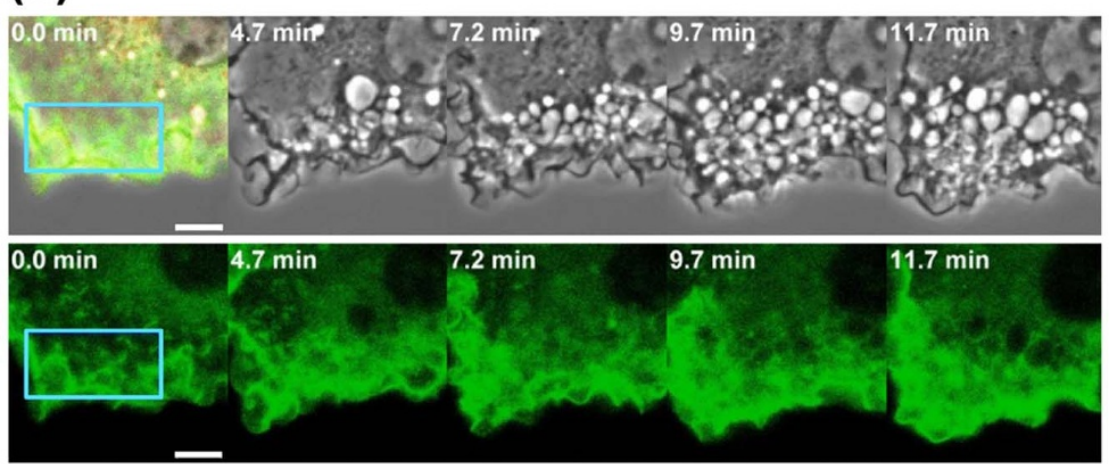

(b)
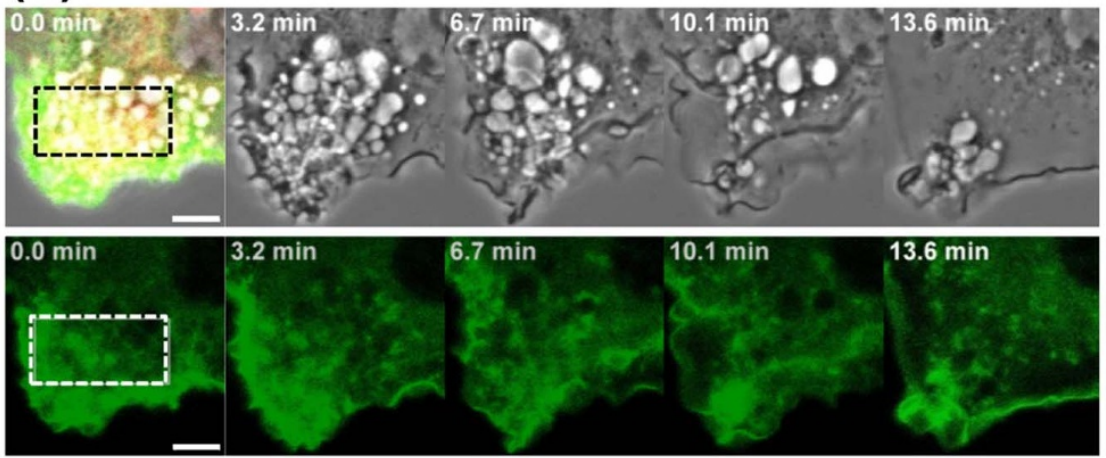

(c)

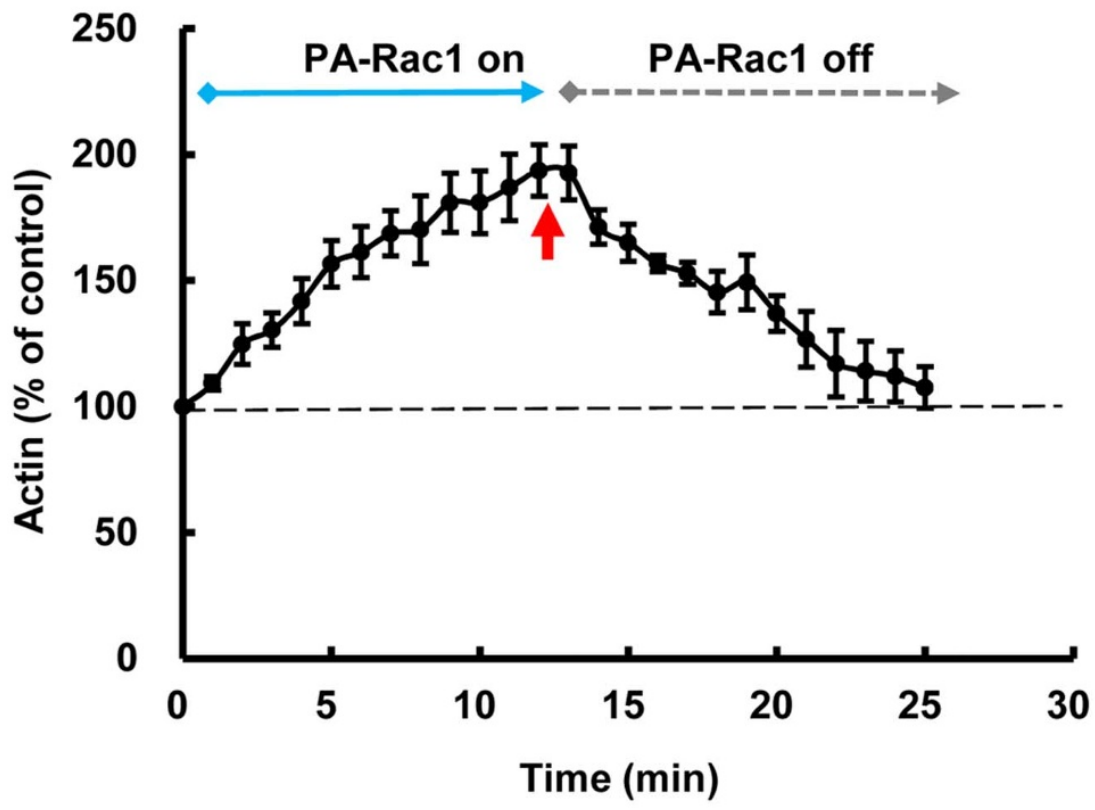

Figure 5 Actin dynamics after PA-Rac1 activation and deactivation. (a) Live-cell images showing actin assembly during PA-Rac1 activation. RAW 264 cells were co-transfected with pTriEx/mCherry-PA-Racl and pYFP- $\beta$-actin. The left top panel shows a merged image of the phase-contrast, mCherry and YFP signals before the photoactivation of PA-Rac1, confirming the expression of mCherry-PA-Racl and YFP- $\beta$-actin. The laser-irradiated area is indicated with a blue rectangle. The other panels show selected time-lapse phase-contrast (upper) and YFP- $\beta$-actin images (lower; green) captured at the indicated times. Time 0 indicates the initiation of photoactivation. The bar indicates $5 \mu \mathrm{m}$. (b) Disassembly of actin from the same cellular region as shown in Fig. 5a after ceasing the $445 \mathrm{~nm}$ laser irradiation. The left top panel shows a merged image of the phase-contrast, mCherry and YFP signals obtained when the irradiation was stopped (time 0 is equivalent to time $11.7 \mathrm{~min}$ in Fig. 5a). The rectangle with broken lines indicates the previously irradiated area in Fig. 5 a. The other panels show selected time-lapse phase-contrast (upper) and YFP- $\beta$-actin images (lower; green) captured at the indicated times. The elapsed times after ceasing irradiation are shown at the top of each panel. The bar indicates $5 \mu \mathrm{m}$. (c) Quantification of the YFP- $\beta$ actin level in the photoactivated area. The YFP- $\beta$-actin signal intensity ratio was calculated and is shown as a $\%$ of the control area. The graph shows the accumulation of YFP- $\beta$-actin in the photoactivated area during PA-Racl photoactivation; and the disappearance of YFP- $\beta$-actin from the photoactivated area after ceasing PA-Racl photoactivation. Data presented are the mean \pm standard error of six independent experiments $(\mathrm{n}=7)$. Time 0 corresponds to initiation of PA-Rac1 photoactivation. Arrow indicates the time point at which irradiation was stopped (12 min after photoactivation). 
(a)

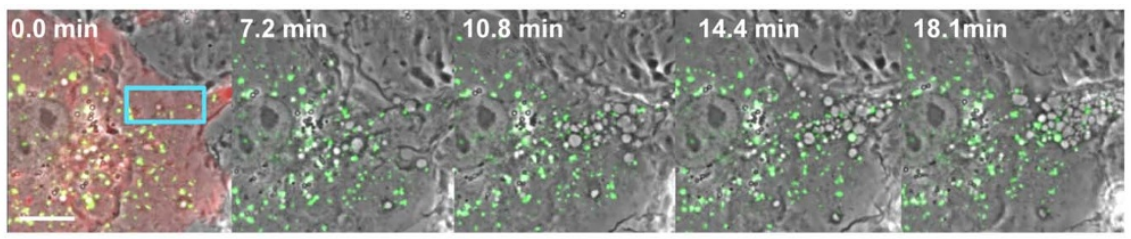

(b)

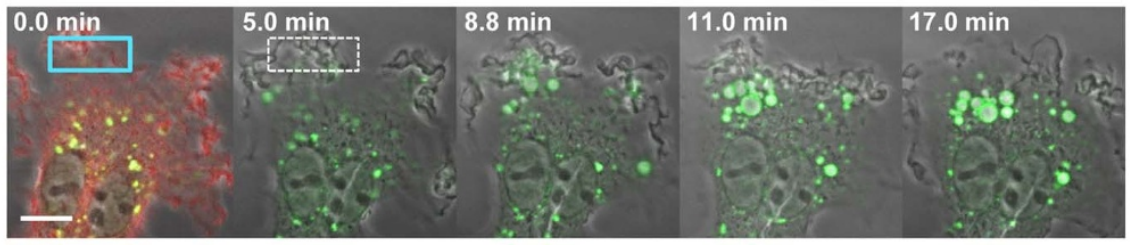

Figure 6 | Persistent PA-Rac1 activation perturbs the recruitment of the Citrine-FYVE domain, a specific probe for PI(3)P, to macropinosomes. (a) RAW 264 cells were co-transfected with pTriEx/mCherry-PA-Racl and pCitrine-FYVE. Photoactivation of PA-Racl was performed by repeated blue laser irradiation for more than $20 \mathrm{~min}$. The first panel shows a merged image of the phase-contrast, mCherry (red) and Citrine (green) signals obtained before the photoactivation of PA-Rac1, confirming the expression of mCherry-PA-Rac1 and Citrine-FYVE. The $445 \mathrm{~nm}$ laser-irradiated area is indicated with a blue rectangle. The other panels show time-lapse merged images of the phase-contrast and Citrine-FYVE fluorescence (green), captured at the indicated times. Time 0 indicates the initiation of photoactivation. The bar indicates $5 \mu \mathrm{m}$. (b) After a 5-min photoactivation period, the laser irradiation was stopped. Note that the 5-min pulse photoactivation efficiently induced the recruitment of PI(3)P probes to newly formed macropinosomes. The bar indicates $5 \mu \mathrm{m}$.
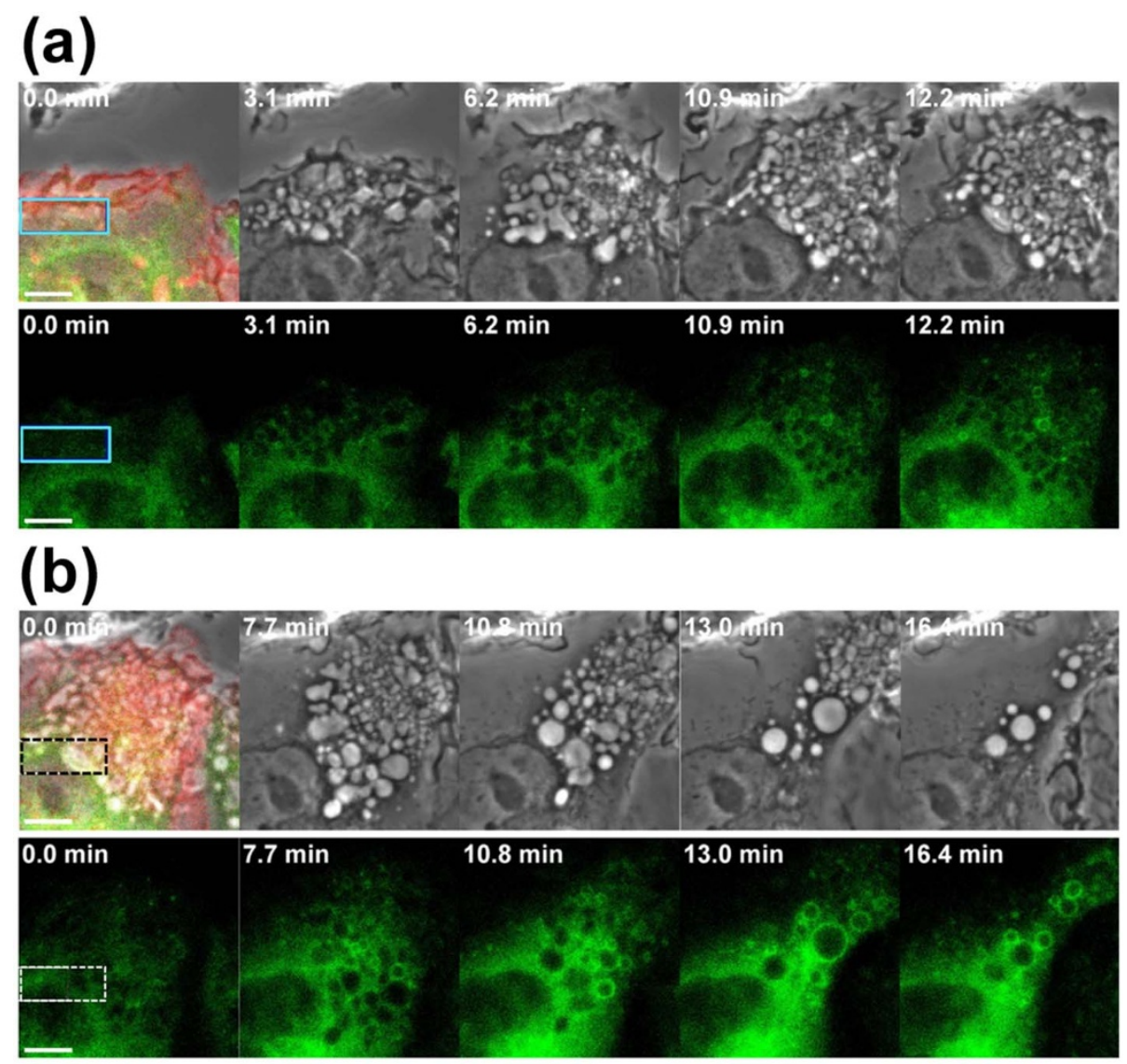

Figure 7 | Live-cell images showing that Rab21 localises to motile macropinosomes, which form after the deactivation of PA-Rac1. (a) RAW264 cells were co-transfected with pTriEx/mCherry-PA-Rac1 and pGFP-Rab21. The blue rectangular area in the cell was repeatedly irradiated using a blue laser for $12.2 \mathrm{~min}$. The top left panel shows a merged image of the phase-contrast, mCherry and GFP fluorescence signals obtained before the photoactivation of PA-Rac1, confirming the expression of mCherry-PA-Rac1 and GFP-Rab21. The other panels show selected time-lapse phase-contrast (upper) and GFPRab21 images (lower; green). Time 0 indicates the initiation of photoactivation. The bars indicate $5 \mu \mathrm{m}$. (b) The recruitment of Rab21 to macropinosomes after PA-Racl deactivation in the same cell as shown in Fig. 7a. The top left panel shows a merged image of the phase-contrast, mCherry and GFP signals obtained when the irradiation was stopped (equivalent to time $12.2 \mathrm{~min}$ in Fig. 7a). The broken line rectangle indicates the previously irradiated area shown in Fig. 7a. The other panels show selected time-lapse phase-contrast (upper) and GFP-Rab21 images (lower; green), captured at the indicated times. Time 0 indicates the time when the photoactivation was stopped. The bar indicates $5 \mu \mathrm{m}$. 
activation of phosphatidylinositol 3-kinase activates guanine nucleotide exchange factors (GEFs) for Racl during growth factor-induced macropinocytosis in fibroblasts ${ }^{1-4}$. However, the upstream activators of Racl during constitutive macropinocytosis in scavenger cells, such as neutrophils, dendritic cells and macrophages, remain unknown. Several molecules have been suggested as downstream effectors of Racl during macropinosome formation, such as PAK1. The overexpression of a constitutively active PAK1 mutant induces macropinosome formation, and overexpression of the PAK1 kinase autoinhibitory domain blocks both growth factor and dominant active Rac1 (Rac1 Q61L)-stimulated macropinocytosis ${ }^{16}$. PAK1 also phosphorylates C-terminal binding protein 1 of the E1A/brefeldin A $\mathrm{ADP}$ ribosylated substrate (CtBP/BARS), a component of the membrane fission machinery, and this phosphorylation is critical for fission of the macropinocytic cup ${ }^{30,31}$. Another candidate molecule, WAVE/ Scar1, which interacts with and activates the Arp2/3 complex ${ }^{32}$, acts downstream of Rac1 and links Racl signalling to actin reorganisation $^{33,34}$. Racl activation stimulates the production of $\mathrm{PI}(4,5) \mathrm{P}_{2}$ by PIP5K and promotes actin polymerisation through the activation of actin-binding proteins ${ }^{23,24,35}$. We have consistently shown that PA-Rac1 activation locally elevates the level of $\mathrm{PI}(4,5) \mathrm{P}_{2}$ and induces the accumulation of actin filaments at the cell periphery under blue laser irradiation. We have previously shown that the level of $\mathrm{PI}(4,5) \mathrm{P}_{2}$ increases during the formation of membrane ruffles and macropinocytic cups, but $\mathrm{PI}(4,5) \mathrm{P}_{2}$ is eliminated when macropinocytic cups close to form macropinosomes ${ }^{22}$. In addition, actin filaments were observed to be disassembled from the bottom of the macropinocytic cups. This suggested that elimination of $\mathrm{PI}(4,5) \mathrm{P}_{2}$ is required for actin filament disassembly and macropinosome closure, suggesting that Racl deactivation is necessary for the efficient closure of macropinosomes.

The photo-manipulation of PA-Racl in RAW264 macrophages enabled us to characterise the role of the Racl molecular switch in the dynamic process of macropinocytosis. This study is the first to show that macropinosome formation can be controlled in live cells by the temporal modulation of a light activatable engineered molecular switch using a conventional confocal laser microscope. This approach could also be used to examine the relationship between Rac1 activation/deactivation and other signalling molecules involved in other cell functions.

\section{Methods}

Chemicals and plasmids. Fluorescein isothiocyanate-dextran, molecular weight 10,000 (FDx), and FM4-64 were purchased from Molecular Probes (Eugene, OR). pTriEx/mCherry-PA-Rac1Q61L and pTriEx/mCherry-PA-Rac1 T17N ${ }^{17,18}$ were obtained from Addgene. Dr. Joel A. Swanson (University of Michigan) kindly provided pCitrine $/ \mathrm{PBD}^{7}$, pCitrine-FYVE ${ }^{7}$, pCitrine-Rab5a ${ }^{7}$, pmCitrine-Rac1Q61L, and pYFP-PLC-PH. Dr. Arwyn T. Jones (EMBL) kindly donated pGFP-Rab21 ${ }^{25}$. The $\mathrm{XhoI} / \mathrm{BamHI}$ fragments of pAcGFP- $\beta$-actin (Clontech) were introduced into the same sites of pEYFP-C1 to produce pEYFP- $\beta$-actin.

Cell culture and transfection. RAW264 mouse macrophage cells (Riken Cell Bank, Tsukuba, Japan) were grown in DMEM containing 10\% foetal bovine serum (FBS) supplemented with $100 \mathrm{U} / \mathrm{ml}$ of penicillin and $0.1 \mathrm{mg} \mathrm{ml}^{-1}$ streptomycin. The cells were maintained at $37^{\circ} \mathrm{C}$ in a humidified $5 \% \mathrm{CO}_{2}$ incubator. Plasmid transfection was performed using the $\mathrm{Neon}^{\mathrm{TM}}$ transfection system (Invitrogen) following the manufacturer's protocol. Briefly, $100 \mu$ of a RAW264 cell suspension $(1.0 \times$ $10^{7}$ cells $/ \mathrm{ml}$ ) in buffer $\mathrm{R}$ was mixed with $1.0-3.0 \mu \mathrm{g}$ of the indicated plasmids and electroporated once at $1,680 \mathrm{~V}$ for $20 \mathrm{~ms}$. The cells were then seeded onto $25-\mathrm{mm}$ circular coverslips in a $35-\mathrm{mm}$ culture dish containing $2 \mathrm{ml}$ of culture medium. At 12-24 hours after transfection, the cells were subjected to live cell imaging.

Photoactivation of PA-Rac1, live cell imaging and data analysis. Transfected RAW264 cells were washed once using pre-warmed Ringer's buffer (RB) [10 mM Hepes- $\mathrm{NaOH}$ (pH 7.2), $155 \mathrm{mM} \mathrm{NaCl}, 5 \mathrm{mM} \mathrm{KCl}, 2 \mathrm{mM} \mathrm{CaCl}, 1 \mathrm{mM} \mathrm{MgCl}$, $2 \mathrm{mM} \mathrm{NaH}_{2} \mathrm{PO}_{4}, 10 \mathrm{mM} \mathrm{D}$-glucose and $0.5 \mathrm{mg} / \mathrm{ml}$ bovine serum albumin (BSA)] and assembled in an RB-filled Attofluor cell chamber (Molecular Probes) on the thermo-controlled stage (Tokai Hit., Shizuoka, Japan). Photoactivation of PA-Rac1 and live cell imaging were performed using an Axio observer $\mathrm{Z} 1$ inverted microscope equipped with a laser scanning unit (LSM700, Zeiss) and Plan-Apochromat 63x/N.A. 1.4 lens under the control of ZEN2009 software (Zeiss). To photoactivate the PA$\mathrm{Rac1}$, the indicated area of RAW264 cells expressing mCherry-PA-Rac1 was repeatedly irradiated using a $5 \mathrm{~mW} 445-\mathrm{nm}$ laser at $1.0-3.0 \%$ power for the indicated intervals of image acquisition. Live cell images were acquired every $15 \mathrm{sec}$ using a $10 \mathrm{~mW} 488-\mathrm{nm}$ laser at $0.5-1.0 \%$ power to obtain GFP, YFP or Citrine images or a $10 \mathrm{~mW} 555-\mathrm{nm}$ laser at $0.5-2.0 \%$ power to obtain mCherry and bright-field phasecontrast or DIC images. The presented image data are representative of at least four independent experiments.

To determine whether bubble-like structures formed by PA-Rac1 photo-manipulation are closed or opened, we added $0.5 \mathrm{mg} \mathrm{ml}^{-1}$ fluorescein dextran (FDx, MW 10,000, Molecular Probe) to the extracellular medium, incubated for $5 \mathrm{~min}$, washed with cold PBS, and then immediately acquired phase-contrast and FDx images. In one experiment, photoactivation was not stopped until FDx was washed out of the medium with cold PBS. In another experiment, photoactivation was turned off $2 \mathrm{~min}$ before washing with PBS.

To identify unclosed macropinocytic cups/pockets, a lipophilic plasma membrane marker, FM4-64 (Molecular Probes) diluted in cold PBS was added to the extracellular medium at a final concentration of $5.0 \mu \mathrm{g} \mathrm{ml}^{-1}$ at different time points $(0 \mathrm{~min}$ $0.5 \mathrm{~min}, 2 \mathrm{~min}$ and $5 \mathrm{~min}$ after photoactivation). Fluorescence images of FM 4-64 were acquired immediately $(<1 \mathrm{~min})$ after the FM4- 64 addition by a confocal laser microscope with $488 \mathrm{~nm}$ excitation and $515 \pm 20 \mathrm{~nm}$ emission. Quantitation of macropinosome closure was carried out by scoring the numbers of FM4-64-negative macropinosomes and FM4-64-positive pre-macropinosomes in the cell. The percentage of FM4-64-negative closed macropinosomes was calculated.

To monitor macropinosome formation and the expression of initial macropinocytic markers, such as actin and $\mathrm{PI}(4,5) \mathrm{P}_{2}$, pYFP-actin or pYFP-PLC- $\delta 1-\mathrm{PH}$ domain was co-transfected with $\mathrm{pTriEx} / \mathrm{mCherry-PA-Rac1Q61L.} \mathrm{To} \mathrm{monitor} \mathrm{macropino-}$ some maturation, Citrine-FYVE or pGFP-Rab21 was co-expressed with mCherryPA-Rac1. To quantify the level of YFP- $\beta$-actin in the photoactivated area, the fluorescence signal intensity ratio was calculated. Briefly, the fluorescence signal intensities from two different areas (photoactivated area and control area) in the YFP $\beta$-actin images were measured using ZEN2009 software, and their ratio (\% of the control) was calculated. The control area which was independent of photoactivation was the same size as the experimental area in the same cell.

Statistical analysis. Data are presented as mean \pm standard error (SE) for the number of experiments indicated in the legends and/or text. Statistical significance was determined by two-tailed unpaired Student's $t$-test. Differences between the analyzed samples were considered significant at $\mathrm{p}<0.05$.

Scanning electron microscopy. RAW264 cells transfected with pmCitrine-fused Rac1Q61L were cultured on cover slips and serum starved for 2 hours. After confirming marked membrane ruffling in Rac1Q61L-expressing cells using fluorescence and phase-contrast microscopy, the cells were fixed with $4 \%$ glutaraldehyde in $0.1 \mathrm{M}$ phosphate buffer, $\mathrm{pH} 7.4$, for 1 hour at $4^{\circ} \mathrm{C}$. The specimens were post-fixed with $1 \% \mathrm{OsO}_{4}$, treated with $1 \%$ tannic acid and processed for scanning EM as previously described ${ }^{11}$. The critical point-dried cells were coated using an osmium plasma coater (OPC40, Nippon Laser \& Electronics) and observed with a Hitachi S900 SEM at $6 \mathrm{kV}$.

1. Swanson, J. A. Shaping cups into phagosomes and macropinosomes. Nat Rev Mol Cell Biol 9, 639-649 (2008).

2. Mercer, J. \& Helenius, A. Virus entry by macropinocytosis. Nat Cell Biol 11, 510-520 (2009)

3. Kerr, M. C. \& Teasdale, R. D. Defining macropinocytosis. Traffic 10, 364-371 (2009).

4. Lim, J. P. \& Gleeson, P. A. Macropinocytosis: an endocytic pathway for internalising large gulps. Immunol Cell Biol 89, 836-843 (2011).

5. Swanson, J. A. \& Watts, C. Macropinocytosis. Trends Cell Biol 5, 424-428 (1995).

6. Kruth, H. S. et al. Macropinocytosis is the endocytic pathway that mediates macrophage foam cell formation with native low density lipoprotein. J Biol Chem 280, 2352-2360 (2005).

7. Yoshida, S., Hoppe, A. D., Araki, N. \& Swanson, J. A. Sequential signaling in plasma-membrane domains during macropinosome formation in macrophages. $J$ Cell Sci 122, 3250-3261 (2009).

8. Swanson, J. A. Phorbol esters stimulate macropinocytosis and solute flow through macrophages. J Cell Sci 94, 135-142 (1989).

9. Racoosin, E. L. \& Swanson, J. A. Macropinosome maturation and fusion with tubular lysosomes in macrophages. J Cell Biol 121, 1011-1020 (1993).

10. Araki, N., Johnson, M. T. \& Swanson, J. A. A role for phosphoinositide 3-kinase in the completion of macropinocytosis and phagocytosis by macrophages. J Cell Biol 135, 1249-1260 (1996).

11. Araki, N., Hatae, T., Furukawa, A. \& Swanson, J. A. Phosphoinositide-3-kinaseindependent contractile activities associated with Fc ganma-receptor-mediated phagocytosis and macropinocytosis in macrophages. J Cell Sci 116, 247-257 (2003).

12. Ridley, A. J., Paterson, H. F., Johnston, C. L., Diekmann, D. \& Hall, A. The small GTP-binding protein rac regulates growth factor-induced membrane ruffling. Cell 70, 401-410 (1992)

13. Bishop, A. L. \& Hall, A. Rho GTPases and their effector proteins. Biochem J 348 Pt 2, 241-255 (2000).

14. Ahram, M. et al. Racl-induced endocytosis is associated with intracellular proteolysis during migration through a three-dimensional matrix. Exp Cell Res 260, 292-303 (2000). 
15. West, M. A., Prescott, A. R., Eskelinen, E. L., Ridley, A. J. \& Watts, C. Rac is required for constitutive macropinocytosis by dendritic cells but does not control its downregulation. Curr Biol 10, 839-848 (2000).

16. Dharmawardhane, S. et al. Regulation of macropinocytosis by $\mathrm{p} 21$-activated kinase-1. Mol Biol Cell 11, 3341-3352 (2000).

17. Wu, Y. I. et al. A genetically encoded photoactivatable Rac controls the motility of living cells. Nature 461, 104-108 (2009).

18. Wu, Y. I., Wang, X., He, L., Montell, D. \& Hahn, K. M. Spatiotemporal control of small GTPases with light using the LOV domain. Methods Enzymol 497, 393-407 (2011).

19. Niggli, V. Regulation of protein activities by phosphoinositide phosphates. Annu Rev Cell Dev Biol 21, 57-79 (2005)

20. Simonsen, A., Wurmser, A. E., Emr, S. D. \& Stenmark, H. The role of phosphoinositides in membrane transport. Curr Opin Cell Biol 13, 485-492 (2001).

21. De Matteis, M. A. \& Godi, A. PI-loting membrane traffic. Nat Cell Biol 6, 487-492 (2004).

22. Araki, N., Egami, Y., Watanabe, Y. \& Hatae, T. Phosphoinositide metabolism during membrane ruffling and macropinosome formation in EGF-stimulated A431 cells. Exp Cell Res 313, 1496-1507 (2007).

23. Ren, X. D. \& Schwartz, M. A. Regulation of inositol lipid kinases by Rho and Rac. Curr Opin Genet Dev 8, 63-67 (1998).

24. Halstead, J. R. et al. Rac controls PIP5K localisation and PtdIns (4,5)P(2) synthesis, which modulates vinculin localisation and neurite dynamics. J Cell Sci $\mathbf{1 2 3}$ 3535-3546 (2010).

25. Egami, Y. \& Araki, N. Dynamic changes in the spatiotemporal localization of Rab21 in live RAW264 cells during macropinocytosis. PLoS One 4, e6689 (2009).

26. Egami, Y. \& Araki, N. Spatiotemporal Localization of Rab20 in Live RAW264 Macrophages during Macropinocytosis. Acta Histochem Cytochem 45, 317-323 (2012).

27. Hamasaki, M., Araki, N. \& Hatae, T. Association of early endosomal autoantigen 1 with macropinocytosis in EGF-stimulated A431 cells. Anat Rec A Discov Mol Cell Evol Biol 277, 298-306 (2004).

28. Araki, N., Hamasaki, M., Egami, Y. \& Hatae, T. Effect of 3-methyladenine on the fusion process of macropinosomes in EGF-stimulated A431 cells. Cell Struct Funct 31, 145-157 (2006).

29. Feliciano, W. D., Yoshida, S., Straight, S. W. \& Swanson, J. A. Coordination of the Rab5 cycle on macropinosomes. Traffic 12, 1911-1922 (2011).

30. Amstutz, B. et al. Subversion of CtBP1-controlled macropinocytosis by human adenovirus serotype 3. EMBO J 27, 956-969 (2008).

31. Liberali, P. et al. The closure of Pak1-dependent macropinosomes requires the phosphorylation of CtBP1/BARS. EMBO J 27, 970-981 (2008).
32. Machesky, L. M. \& Insall, R. H. Scarl and the related Wiskott-Aldrich syndrome protein, WASP, regulate the actin cytoskeleton through the Arp2/3 complex. Curr Biol 8, 1347-1356 (1998).

33. Miki, H., Suetsugu, S. \& Takenawa, T. WAVE, a novel WASP-family protein involved in actin reorganization induced by Rac. EMBO J 17, 6932-6941 (1998).

34. Miki, H., Yamaguchi, H., Suetsugu, S. \& Takenawa, T. IRSp53 is an essential intermediate between Rac and WAVE in the regulation of membrane ruffling. Nature 408, 732-735 (2000).

35. Hartwig, J. H. et al. Thrombin receptor ligation and activated Rac uncap actin filament barbed ends through phosphoinositide synthesis in permeabilized human platelets. Cell 82, 643-653 (1995).

\section{Acknowledgements}

The authors would like to thank Dr. Joel A. Swanson (University of Michigan) for providing plasmids and advice and Dr. Arwyn T. Jones (EMBL) for kindly donating pGFP-Rab21. The authors would also like to thank Dr. Katsuya Miyake, Dr. Takuma Kato, Ms. Saya Kitashiro, Ms. Yurie Ishikawa, Ms. Yuka Ikeda, Ms. Yukiko Iwabu, and Mr. Kazuhiro Yokoi in our laboratory for their assistance and advice. This study was primarily supported by funding from JSPS KAKENHI grants No. 23390039 and No.24659087 to N.A and the MIU foundation, and partial funding was received through grants No. 23890144 to M.F., No. 80534510 to K.K. and No. 22790188 to Y.E. from JSPS.

\section{Author contributions}

M.F., K.K. and N.A. conceived the study and designed the experiments; M.F. and K.K. performed the imaging experiments and collected data. Y.E. contributed to the molecular cloning and transfection experiments. M.F. and N.A. wrote the main manuscript text, and all authors reviewed the manuscript.

\section{Additional information}

Supplementary information accompanies this paper at http://www.nature.com/ scientificreports

Competing financial interests: The authors declare no competing financial interests. How to cite this article: Fujii, M., Kawai, K., Egami, Y. \& Araki, N. Dissecting the roles of Racl activation and deactivation in macropinocytosis using microscopic photo-manipulation. Sci. Rep. 3, 2385; DOI:10.1038/srep02385 (2013).

(c) (i) $(9)$ This work is licensed under a Creative Commons Attribution(1) No visit http://creativecommons.org/licenses/by-nc-nd/3.0 